Results: 109 patients with metastatic ChRCC were identified including 29 with SF. Median TTR post nephrectomy was shorter for patients with vs. without SF $(2.7$ months [95\% CI: 0.7, 6.9] vs. 48.8 months [95\% CI: 30.8, 80.7], log-rank p<0.001). Median TTF on first line therapy was shorter for patients with vs. without SF $(1.8$ months [95\% CI: 0.9, 2.7] vs. 8.0 months [95\% CI: 5.1, 13.0]; log-rank $\mathrm{p}<0.001$ ). No responses were observed in 6 patients treated with nivolumab including 4 with SF. Median OS was inferior for patients with vs. without SF (38 months vs.7.5 months, HR 4.7 [95\% CI: 2.7, 8.2], p<0.001). NGS, performed in 22 patients, showed that $64 \%$ and 45\% harbored TP53 and PTEN alterations, respectively. MSI high status was identified in 3 patients.

Conclusions: Metastatic ChRCC patients with SF have worse outcomes compared to those without SF. Median TTR < 3 months for this subgroup supports close surveillance following nephrectomy for localized tumors. Lack of benefit with various systemic regimens warrants studying underlying biology and investigating novel agents.

\section{Micro abstract}

We studied cancer specifics outcomes of 109 patients with metastatic chromophobe RCC with and without sarcomatoid features. We found that patients with metastatic sarcomatoid chromophobe renal cell carcinoma had uniformly poor outcomes with a short time to recurrence with metastatic disease post-nephrectomy, limited response to systemic therapy and poor overall survival compared to those without sarcomatoid features.

\section{Keywords}

Chromophobe; Renal cell carcinoma; Genomics; Non-clear cell renal cell carcinoma; Sarcomatoid

\section{Background:}

Kidney cancer is the sixth most common malignancy worldwide with an incidence of 64,000 new cases in the United States in 2017(1). It is well known that this is a diverse disease comprised of multiple histological variants with different clinical outcomes and molecular features (2) (3). Chromophobe RCC (ChRCC) is one of the less common RCC subtypes representing $\sim 5-10 \%$ of all cases (2) (4). In the non-metastatic setting it is known to be associated with favorable outcomes with 10-year survival rates of $80-90 \%$ and low risk of metastasis (5). In the metastatic setting favorable responses to VEGF-TKI agents and mTOR inhibitors were reported in patients with metastatic ChRCC receiving treatment on phase II clinical trials (6-8). Given the relative rarity of this disease, dedicated prospective clinical trials in ChRCC have not been reported to date.

Sarcomatoid differentiation, a feature on histopathologic review, can occur in any subtype of RCC, automatically implies high-grade disease, and as such is well described as an adverse feature across RCC variants. Several series report its negative prognostic effect in patients with localized disease undergoing nephrectomy in curative intent(9). Evidence in the metastatic setting is limited to small retrospective series analyzing patients receiving systemic therapy across a variety of RCC variants $(10,11)$. Incidence of sarcomatoid features in ChRCC appears to be low, estimated at 2-11\% across series (12-15). Data for 
metastatic ChRCC with sarcomatoid differentiation is limited but suggests poor outcome compared to patients without such features(16). Recognizing the apparent discrepancy in outcomes between ChRCC in general and this particular variant, we sought to carry out a retrospective analysis of patients managed at Memorial Sloan Kettering Cancer Center (MSKCC) in the metastatic setting, evaluating their clinical presentation, systemic therapy outcomes and genomic features based on the presence vs. absence of sarcomatoid features.

\section{Materials and methods:}

We obtained institutional review board approval to identify patients with non-clear cell RCC within the institution database. Included were all patients with metastatic ChRCC who were evaluated in Medical Oncology clinic at MSKCC prior to initiating first line systemic therapy. Subjects were identified via an automated search of pathology reports within the institutional medical record using the search terms "Chromophobe" and "Renal cell carcinoma" for reports issued between 01/01/2000 and 09/27/2017. Individual charts were then manually reviewed in detail to confirm eligibility and compile data. Eligible patients for the study were $>18$ years old, pathologically confirmed at MSKCC to have ChRCC histology, had confirmed metastatic disease as per MSKCC Radiology evaluation and were evaluated by Medical Oncology at our institution prior to starting first line systemic therapy. We recorded demographics, clinical features, including International Metastatic Database Consortium (IMDC) risk status, treatment details and survival outcomes.

\section{Pathology review and genomics:}

Pathology slides for all patients were reviewed by a dedicated MSKCC genitourinary pathologist. Sarcomatoid differentiation in the background of ChRCC was defined histologically by the presence of a malignant spindle cell component abruptly co-existed with an epithelial component with typical appearance of ChRCC. For this study, any extent of sarcomatoid differentiation qualified as 'sarcomatoid features'. We recorded the primary tumor size and the extent of sarcomatoid differentiation if included in the pathology report.

In a subset of patients, next generation sequencing (NGS) was performed using MSKIMPACT $^{\mathrm{TM}}$ a custom pull-down assay with germline comparison from peripheral blood samples that achieves hybridization capture with target-specific probes from exons of at least 341 cancer associated genes (17). This assay identifies mutations, alterations in copy number, and reports on the microsatellite instability (MSI) sensor score and tumor mutational burden (TMB). MSI-sensor score $\geq 10$ was previously shown to reliably define MSI high (MSI-H) status phenotype, while scores $\geq 3$ to 10 and $<3$ are considered intermediate and low respectively (18). For copy number alterations being frequent somatic event in ChRCC $(19,20)$, the fraction of copy-number altered genome (FCNAg) was calculated by considering the average proportion of each chromosome that was affected by somatic copy-number events, weighed on the 22 autosomal chromosomes(21).

\section{Statistical considerations:}

Patients demographics and baseline clinical features were summarized by descriptive statistics. Time to recurrence (TTR) was calculated for patients who had initially presented 
with localized disease and underwent nephrectomy in curative intent. For these, TTR was defined as time from date of nephrectomy to first radiographic evidence of metastatic recurrence, and log-rank analysis was used for comparison between patients with and without sarcomatoid differentiation. Overall survival (OS) was calculated using the KaplanMeier method from the patient's first date of evaluation at MSKCC by Medical Oncology for metastatic disease until death; patients who were still alive at the data cutoff were censored at date of last follow-up. Analysis of treatment outcomes was limited to those patients who initiated first line systemic therapy at MSKCC. Time to treatment failure (TTF), was calculated as the interval between the date of initiating therapy and the date of radiographic progression, drug cessation for any other reason, or death, whichever occurred first; TTF was censored at time of the last follow-up date for those remaining on therapy. Radiographic progression and treatment discontinuation were documented per the treating oncologist's discretion. The association between FCNAg and MSI-sensor scores was evaluated using Spearman's rank correlation coefficient test. The cutoff date for follow-up and survival status was October 1, 2017.

\section{Results:}

\section{Baseline features:}

The institutional electronic database search returned with 950 entries between 2002 and 2017, out of which 109 patients with newly diagnosed metastatic ChRCC were identified. Baseline patient characteristics are summarized on Table 1. Sarcomatoid differentiation was present in 29 patients (27\%), was extensive in 20 patients (69\%) and focal in two patients (7\%). The Median age at diagnosis with metastatic disease for all patients was 58 years (Range, 40-84 years), this was similar among patients with and without sarcomatoid differentiation. Presentation with De Novo metastatic disease and systemic symptoms of kidney cancer were significantly more common in patients whose tumors harbored sarcomatoid elements compared to those without it (48 \% vs. 19\% [Fisher's exact test, $\mathrm{P}=0.002$ ] and $48 \%$ vs. $10 \%$ [Fisher's exact test, $\mathrm{P}<0.0001$ ]) respectively. Similarly, these patients were more likely to be categorized as IMDC intermediate or poor risk (Fisher's exact test, $\mathrm{P}<0.001)$. Distribution of metastatic sites was notable for high incidence of pulmonary metastases in patients with sarcomatoid features (62\% vs. 24\%; Fisher's exact test, $\mathrm{P}<0.001)$.

\section{Clinical course and treatment outcomes}

Median TTR post-nephrectomy for 80 patients who had initially presented with nonmetastatic disease was 35.5 months (95\% CI: 23.4, 58.3). TTR was significantly longer for 65 patients without sarcomatoid differentiation compared to 15 patients with sarcomatoid differentiation (48.8 months [95\% CI: 30.8, 80.7] vs. 2.7 months [95\% CI: 0.7, 6.9], logrank $\mathrm{p}<0.001$ ) (Figure 1).

A total of 52 patients initiated first line therapy at MSKCC including 35 without and 17 with sarcomatoid differentiation. Outcomes of systemic therapy are summarized on Table 2. First line TTF across all 52 patients was 5.1 months (95\% CI: 2.7, 8.0) and different significantly depending on presence/absence of sarcomatoid features. For 35 patients without sarcomatoid 
differentiation, median TTF was 8.0 months (95\% CI: 5.1, 13.0); by comparison, 17 sarcomatoid patients achieved a median TTF of 1.8 months (95\% CI: 0.9, 2.7) (log-rank $\mathrm{p}<0.001)$. Antitumor effect remained poor with subsequent lines of therapy for the latter group. Figure 2 visualizes individual courses of patients with sarcomatoid differentiation and summarizes treatment duration for all lines received. One patient with favorable risk IMDC status and focal sarcomatoid differentiation was treated with first line temsirolimus for 14.4 months which was discontinued due to pulmonary toxicity. Nivolumab was administered in six patients (four with sarcomatoid, two without sarcomatoid differentiation) with a median TTF of 2.6 months (Range, 0.6-8.7 months). Imaging evaluation was available in four out of the six patients which showed no disease response. Details on all systemic agents used in different lines of therapy are provided on Table S1, S2 and S3.

Median OS for all 109 patients from the date of first evaluation at our center for management of metastatic disease was 25 months (95\% CI: 12, 33), with survival rate of $62.4 \%$ at 12 months (95\% CI: 51.8, 71.3). The median follow-up time for survivors was 14 months. Median OS was significantly longer for patients without sarcomatoid differentiation compared to those with sarcomatoid differentiation (38 months vs.7.5 months, HR 4.7, [95\% CI: 2.7, 8.2], log-rank p<0.001) (Figure 3). Survival rates for patients without and with sarcomatoid differentiation at 12 months were $74.9 \%$ and $19.7 \%$ respectively (Table S4).

OS varied significantly by IMDC risk stratification (log-rank $\mathrm{p}<0.001)$, with a median OS of 62.2 months (95\% CI: 32.7, not estimated) for 42 favorable-risk patients, 12.2 months (95\% CI: $6.9,24.1)$ for 42 intermediate risk patients and 4.9 months (95\% CI: $2.8,9.9)$ for 16 poor risk patients (Figure S1). The difference in survival outcomes by IMDC score was consistent among patients without sarcomatoid differentiation (log-rank $\mathrm{p}<0.001$ ), however for the sarcomatoid group, almost all patients (27 of 29) were in the intermediate and poor IMDC risk groups, and OS did not differ between those two risk groups (log-rank $\mathrm{p}=0.33$ ) (Figure S2). Two patients with sarcomatoid differentiation were IMDC favorable risk and died at 52.1 and 15.7 months from initial evaluation at MSKCC.

\section{Molecular analysis:}

NGS with IMPACT ${ }^{\mathrm{TM}}$ was performed in 22 patients including 30 samples from 6 patients with and 16 without sarcomatoid differentiation. Most common mutations were TP53 followed by PTEN in 14(64\%) and 10(45\%) patients respectively, and these didn't differ between the 2 groups. (Table S5) (Figure 4). Germline testing was performed in 12 patients and 1 patient without sarcomatoid differentiation had an underlying APC germline mutation. Median TMB for all 22 patients was 2.8 mutation/megabase (Range, 0-17.9), this was 2.8 mutation/megabase (Range, 0-17.9) for patients without sarcomatoid differentiation and 3.7 mutation/megabase (Range, 1.6-8.5) for those with sarcomatoid differentiation. Median MSI-sensor score across all samples was 1.5 (Range, 0-17.2). Samples from three and four patients without sarcomatoid differentiation displayed high MSI-sensor score (14\%) and intermediate scores (18\%), respectively. All samples from patients with sarcomatoid differentiation displayed low MSI-sensor score. None of the patients with MSI-H phenotype were treated with immune-checkpoint inhibitors at the time of report. There was a strong 
association between higher MSI-sensor scores and FCNAg (Spearman's rank correlation coefficient $=0.61, \mathrm{P}<0.001)($ Figure 5).

\section{Discussion:}

Several large studies have indicated that $\mathrm{ChRCC}$ is a disease with favorable survival outcomes and low risk of metastasis $(5,13)$. Although the presence of certain high risk pathological features was shown to associate with poor outcomes including sarcomatoid features $(14,15,22)$, the number of patients with sarcomatoid differentiation in those studies was low limiting the degree of detail these could provide on clinical outcomes, particularly in the metastatic setting. In the present study we examined outcomes for the largest cohort of metastatic ChRCC reported to date, 109 patients including 29 cases with sarcomatoid differentiation. We found prognostic significance for the presence of sarcomatoid differentiation which reflected striking differences in clinical presentation, outcomes with systemic therapy and OS.

Several findings speak to the aggressive phenotype of this ChRCC variant. A higher proportion of patients with sarcomatoid ChRCC presented with de novo metastatic disease. For those who had initially diagnosed with non-metastatic disease, underwent nephrectomies and subsequently recurred, the interval from surgery to metastatic recurrence was strikingly shorter if sarcomatoid features were present in the nephrectomy specimen, with a median time of only 2.7 months $(95 \%$ CI: $0.7,6.9)$. This data should be taken into consideration when planning active surveillance for such patients.

The OS of 80 patients with metastatic ChRCC without sarcomatoid differentiation in our series was comparable to other reported series with a median OS of 38 months. Similar to prior reports we demonstrated that the IMDC risk model is applicable to this histologic variant of RCC in these patients treated in the era of targeted therapy(23). Conversely, most of cases harboring sarcomatoid features were categorized as intermediate/poor risk, and their outcome was uniformly poor with median OS of 7.5 months (95\% CI: 4.2, 10.7) and only $20 \%$ of patients alive at 1 year. Lauer et (16) previously examined clinico-pathological features of 14 patients with sarcomatoid ChRCC and reported a similar short median survival time of 8 weeks with 9 patients (64\%) dead of disease within 6 months.

With regards to outcomes to first line systemic therapy, ASPEN (6) and ESPN (7) were randomized phase II clinical trials that each compared first line everolimus versus sunitinib in patients with metastatic non-clear cell RCC. While these are the largest prospective trials ever reported for non-clear cell RCC and included ChRCC patients, these weres enrolled amongst various other non-clear RCC variants, and the primary efficacy analyses were conducted across these heterogeneous populations. Both trials together only included a total of 28 patients with metastatic ChRCC. Secondary subset analyses were conducted but must be interpreted cautiously considering the small sample sizes of such groups. Median progression free survival (PFS) for sunitinib and everolimus in patients with metastatic ChRCC treated on ASPEN were 5.5 months (80\% CI: 3.2-19.7) and 11.4 months (80\% CI: 5.7-19.4), respectively. ESPN reported a median PFS of 8.9 months (80\% CI: 2.9-20.1) for sunitinib and not estimated for everolimus. Grossly, such findings are comparable to a 
median TTF of 8.0 months for 37 patients with metastatic ChRCC without sarcomatoid features reported here. In contrast, TTF was notably shorter among patients with sarcomatoid differentiation for whom we determined a median first-line TTF of 1.8 months across different systemic agents. Prospective phase II clinical trials have investigated cytotoxic therapy and targeted therapy in patients with metastatic sarcomatoid RCC with multiple histologies including combinations of Ifosfamide/Doxorubicin(24), Gemcitabine/ Doxorubicin(25) and Gemcitabine/Sunitinib(26) with poor outcomes overall, although it is noteworthy that one out of three patients with metastatic ChRCC with sarcomatoid differentiated who were treated with Gemcitabine/Doxorubicin achieved a partial response. Similarly, results from retrospective studies on the use of targeted therapies in patients with metastatic RCC with sarcomatoid differentiated which included patients with chromophobe histology (10) (27) have demonstrated poor overall outcomes.

The advent of immune-checkpoint inhibitors has revolutionized the treatment paradigms of patients with clear cell RCC both in the first and second line settings. Recently two retrospective studies (28) (29) reported outcomes of nivolumab in patients across various non-clear cell RCC histologies. These included a total of 15 patients with metastatic ChRCC, and none achieved radiological objective responses. In our series, 6 patients were treated with nivolumab including four with sarcomatoid differentiation with a TTF of 2.6 months (Range, 0.6-8.7 months) and no radiological objective responses were observed. Prior studies have demonstrated that ChRCC is characterized with low PD-L1 expression(30) which may explain some of the possible reasons for lack of response.

For 22 patients in our series who underwent NGS, we found that TP53 and PTEN were the most frequently mutated genes in $64 \%$ and $45 \%$ of cases respectively, and these were not different between patients with and without sarcomatoid features. Similarly, the TCGA database(19) included 66 patients with ChRCC and identified TP53 and PTEN as the most frequently mutated genes in $32 \%$ and $9 \%$ of cases respectively. Another recent study(20) examined genomic alteration in primary tumors from 144 cases including patients from the TCGA cohort and identified that TP53, PTEN mutations and imbalanced chromosomal duplication were more frequent genomic events in patients with metastatic ChRCC compared to non-metastatic patients, and the presence of any of these three was found to associate with poor outcomes. Another important finding in our study is that MSI-H phenotype based on high MSI-sensor scores was identified in samples from 3 patients without sarcomatoid differentiation (14\% of cohort with NGS). In a study by Midda el al (18) reporting on MSI statuses across 12,288 patients with 66 principle cancers utilizing the MSI-sensor score method, 1 of 349 patients with RCC were MSI-H (0.29\%) and as per our correspondence with the authors, this patient had ChRCC. One of the explanation for this observation could be the association between higher copy number alterations with higher MSI-sensor scores as demonstrated in our study. Therapeutic effects of targeted immunotherapy in ChRCC with intermediate/high MSI scores certainly is of interest for future investigation.

The limitations of our study may include the retrospective nature, the use of heterogeneous therapeutic agents, and the use TTF for the assessment of systemic therapy effect instead of PFS, making it more difficult to put our findings in context with prior reports. 


\section{Conclusions:}

The presence of sarcomatoid differentiation in ChRCC transforms disease biology to a high grade aggressive phenotype with uniformly poor outcomes, including early recurrence after nephrectomies performed in curative intent and a lack of efficacy with standard targeted therapies in the metastatic setting. These findings highlight this ChRCC variant as its own clinical entity and warrants further study of the underlying disease biology and development of dedicated management strategies.

\section{Supplementary Material}

Refer to Web version on PubMed Central for supplementary material.

\section{Acknowledgments}

Funding: Precision Immunotherapy Kidney Cancer Program, Tuttle Rare Kidney Cancer Fund, Center Core Grant P30 CA008748 from the National Cancer Institute.

\section{References}

1. Siegel RL, Miller KD, Jemal A. Cancer Statistics, 2017. CA Cancer J Clin. 2017;67(1):7-30. [PubMed: 28055103]

2. Moch H, Cubilla AL, Humphrey PA, Reuter VE, Ulbright TM. The 2016 WHO Classification of Tumours of the Urinary System and Male Genital Organs-Part A: Renal, Penile, and Testicular Tumours. Eur Urol. 2016;70(1):93-105. [PubMed: 26935559]

3. Cancer Genome Atlas Research N. Comprehensive molecular characterization of clear cell renal cell carcinoma. Nature. 2013;499(7456):43-9. [PubMed: 23792563]

4. Reuter VE, Presti JC Jr. Contemporary approach to the classification of renal epithelial tumors. Semin Oncol. 2000;27(2):124-37. [PubMed: 10768592]

5. Cheville JC, Lohse CM, Zincke H, Weaver AL, Blute ML. Comparisons of outcome and prognostic features among histologic subtypes of renal cell carcinoma. Am J Surg Pathol. 2003;27(5):612-24. [PubMed: 12717246]

6. Armstrong AJ, Halabi S, Eisen T, Broderick S, Stadler WM, Jones RJ, et al. Everolimus versus sunitinib for patients with metastatic non-clear cell renal cell carcinoma (ASPEN): a multicentre, open-label, randomised phase 2 trial. Lancet Oncol. 2016;17(3): 378-88. [PubMed: 26794930]

7. Tannir NM, Jonasch E, Albiges L, Altinmakas E, Ng CS, Matin SF, et al. Everolimus Versus Sunitinib Prospective Evaluation in Metastatic Non-Clear Cell Renal Cell Carcinoma (ESPN): A Randomized Multicenter Phase 2 Trial. Eur Urol. 2016;69(5):866-74. [PubMed: 26626617]

8. Motzer RJ, Barrios CH, Kim TM, Falcon S, Cosgriff T, Harker WG, et al. Phase II randomized trial comparing sequential first-line everolimus and second-line sunitinib versus first-line sunitinib and second-line everolimus in patients with metastatic renal cell carcinoma. J Clin Oncol. 2014;32(25): 2765-72. [PubMed: 25049330]

9. Leibovich BC, Lohse CM, Cheville JC, Zaid HB, Boorjian SA, Frank I, et al. Predicting Oncologic Outcomes in Renal Cell Carcinoma After Surgery. Eur Urol. 2018;73(5):772-80. [PubMed: 29398265]

10. Molina AM, Tickoo SK, Ishill N, Trinos MJ, Schwartz LH, Patil S, et al. Sarcomatoid-variant renal cell carcinoma: treatment outcome and survival in advanced disease. American journal of clinical oncology. 2011;34(5):454-9. [PubMed: 21127411]

11. Golshayan AR, George S, Heng DY, Elson P, Wood LS, Mekhail TM, et al. Metastatic sarcomatoid renal cell carcinoma treated with vascular endothelial growth factor-targeted therapy. J Clin Oncol. 2009;27(2):235-41. [PubMed: 19064974] 
12. de Peralta-Venturina M, Moch H, Amin M, Tamboli P, Hailemariam S, Mihatsch M, et al. Sarcomatoid differentiation in renal cell carcinoma: a study of 101 cases. Am J Surg Pathol. 2001 ; 25(3):275-84. [PubMed: 11224597]

13. Volpe A, Novara G, Antonelli A, Bertini R, Billia M, Carmignani G, et al. Chromophobe renal cell carcinoma (RCC): oncological outcomes and prognostic factors in a large multicentre series. BJU Int. 2012;110(1):76-83. [PubMed: 22044519]

14. Amin MB, Paner GP, Alvarado-Cabrero I, Young AN, Stricker HJ, Lyles RH, et al. Chromophobe renal cell carcinoma: histomorphologic characteristics and evaluation of conventional pathologic prognostic parameters in 145 cases. Am J Surg Pathol. 2008;32(12):1822-34. [PubMed: 18813125]

15. Przybycin CG, Cronin AM, Darvishian F, Gopalan A, Al-Ahmadie HA, Fine SW, et al. Chromophobe renal cell carcinoma: a clinicopathologic study of 203 tumors in 200 patients with primary resection at a single institution. Am J Surg Pathol. 2011;35(7):962-70. [PubMed: 21602658]

16. Lauer SR, Zhou M, Master VA, Osunkoya AO. Chromophobe renal cell carcinoma with sarcomatoid differentiation: a clinicopathologic study of 14 cases. Anal Quant Cytopathol Histpathol. 2013;35(2):77-84. [PubMed: 23700716]

17. Cheng DT, Mitchell TN, Zehir A, Shah RH, Benayed R, Syed A, et al. Memorial Sloan KetteringIntegrated Mutation Profiling of Actionable Cancer Targets (MSK-IMPACT): A Hybridization Capture-Based Next-Generation Sequencing Clinical Assay for Solid Tumor Molecular Oncology. J Mol Diagn. 2015;17(3):251-64. [PubMed: 25801821]

18. Middha S, Zhang L, Nafa K, Jayakumaran G, Wong D, Kim HR, et al. Reliable PanCancer Microsatellite Instability Assessment by Using Targeted Next-Generation Sequencing Data. JCO precision oncology. 2017;2017.

19. Davis CF, Ricketts CJ, Wang M, Yang L, Cherniack AD, Shen H, et al. The somatic genomic landscape of chromophobe renal cell carcinoma. Cancer Cell. 2014;26(3):319-30. [PubMed: 25155756]

20. Casuscelli J, Weinhold N, Gundem G, Wang L, Zabor EC, Drill E, et al. Genomic landscape and evolution of metastatic chromophobe renal cell carcinoma. JCI Insight. 2017;2(12).

21. Zack TI, Schumacher SE, Carter SL, Cherniack AD, Saksena G, Tabak B, et al. Pancancer patterns of somatic copy number alteration. Nature genetics. 2013;45(10): 1134-40. [PubMed: 24071852]

22. Cheville JC, Lohse CM, Sukov WR, Thompson RH, Leibovich BC. Chromophobe renal cell carcinoma: the impact of tumor grade on outcome. Am J Surg Pathol. 2012;36(6):851-6. [PubMed: 22367296]

23. Kroeger N, Xie W, Lee JL, Bjarnason GA, Knox JJ, Mackenzie MJ, et al. Metastatic non-clear cell renal cell carcinoma treated with targeted therapy agents: characterization of survival outcome and application of the International mRCC Database Consortium criteria. Cancer. 2013;119(16):2999_ 3006. [PubMed: 23696129]

24. Escudier B, Droz JP, Rolland F, Terrier-Lacombe MJ, Gravis G, Beuzeboc P, et al. Doxorubicin and ifosfamide in patients with metastatic sarcomatoid renal cell carcinoma: a phase II study of the Genitourinary Group of the French Federation of Cancer Centers. J Urol. 2002;168(3):959-61. [PubMed: 12187199]

25. Haas NB, Lin X, Manola J, Pins M, Liu G, McDermott D, et al. A phase II trial of doxorubicin and gemcitabine in renal cell carcinoma with sarcomatoid features: ECOG 8802. Med Oncol. 2012;29(2):761-7. [PubMed: 21298497]

26. Michaelson MD, McKay RR, Werner L, Atkins MB, Van Allen EM, Olivier KM, et al. Phase 2 trial of sunitinib and gemcitabine in patients with sarcomatoid and/or poor-risk metastatic renal cell carcinoma. Cancer. 2015;121(19):3435-43. [PubMed: 26058385]

27. Voss MH, Bastos DA, Karlo CA, Ajeti A, Hakimi AA, Feldman DR, et al. Treatment outcome with mTOR inhibitors for metastatic renal cell carcinoma with nonclear and sarcomatoid histologies. Ann Oncol. 2014;25(3):663-8. [PubMed: 24458473]

28. McKay RR, Bosse D, Xie W, Wankowicz SAM, Flaifel A, Brandao R, et al. The Clinical Activity of PD-1/PD-L1 Inhibitors in Metastatic Non-Clear Cell Renal Cell Carcinoma. Cancer Immunol Res. 2018. 
29. Koshkin VS, Barata PC, Zhang T, George DJ, Atkins MB, Kelly WJ, et al. Clinical activity of nivolumab in patients with non-clear cell renal cell carcinoma. Journal for immunotherapy of cancer. 2018;6(1):9. [PubMed: 29378660]

30. Choueiri TK, Fay AP, Gray KP, Callea M, Ho TH, Albiges L, et al. PD-L1 expression in nonclearcell renal cell carcinoma. Ann Oncol. 2014;25(11):2178-84. [PubMed: 25193987] 


\section{Clinical practice points:}

- $\quad$ ChRCC is a subtype of kidney cancer which is generally known to be associated with favorable outcomes although the presence of certain high risk pathological features such as sarcomatoid differentiation was shown to be associated with poor outcomes.

- We examined clinical outcomes and genomics features on the largest series of metastatic ChRCC $(n=109)$ reported to date, comparing outcomes between patients with and without sarcomatoid features.

- We found that patients with metastatic ChRCC with sarcomatoid features had significantly worse outcomes across all clinical endpoints considered in our analysis including significantly shorter time to recurrence with metastatic disease postnephrectomy, limited response to systemic therapy and poor overall survival compared to those without sarcomatoid features.

- $\quad$ Median TTR $<3$ months with metastatic disease in patients with ChRCC with sarcomatoid features after undergoing nephrectomy in curative intent, supports close surveillance following nephrectomy for localized tumors in this variant.

- $\quad$ Genomic features were examined in a subset of patient with NGS ( $\mathrm{n}=2)$ and we found that harbored TP53 and PTEN mutations were the most frequently mutated genes in 14(64\%) and 10(45\%) of patients respectively which to previous. We also found that MSI high phenotype was identified in 3 patients $(14 \%)$. 


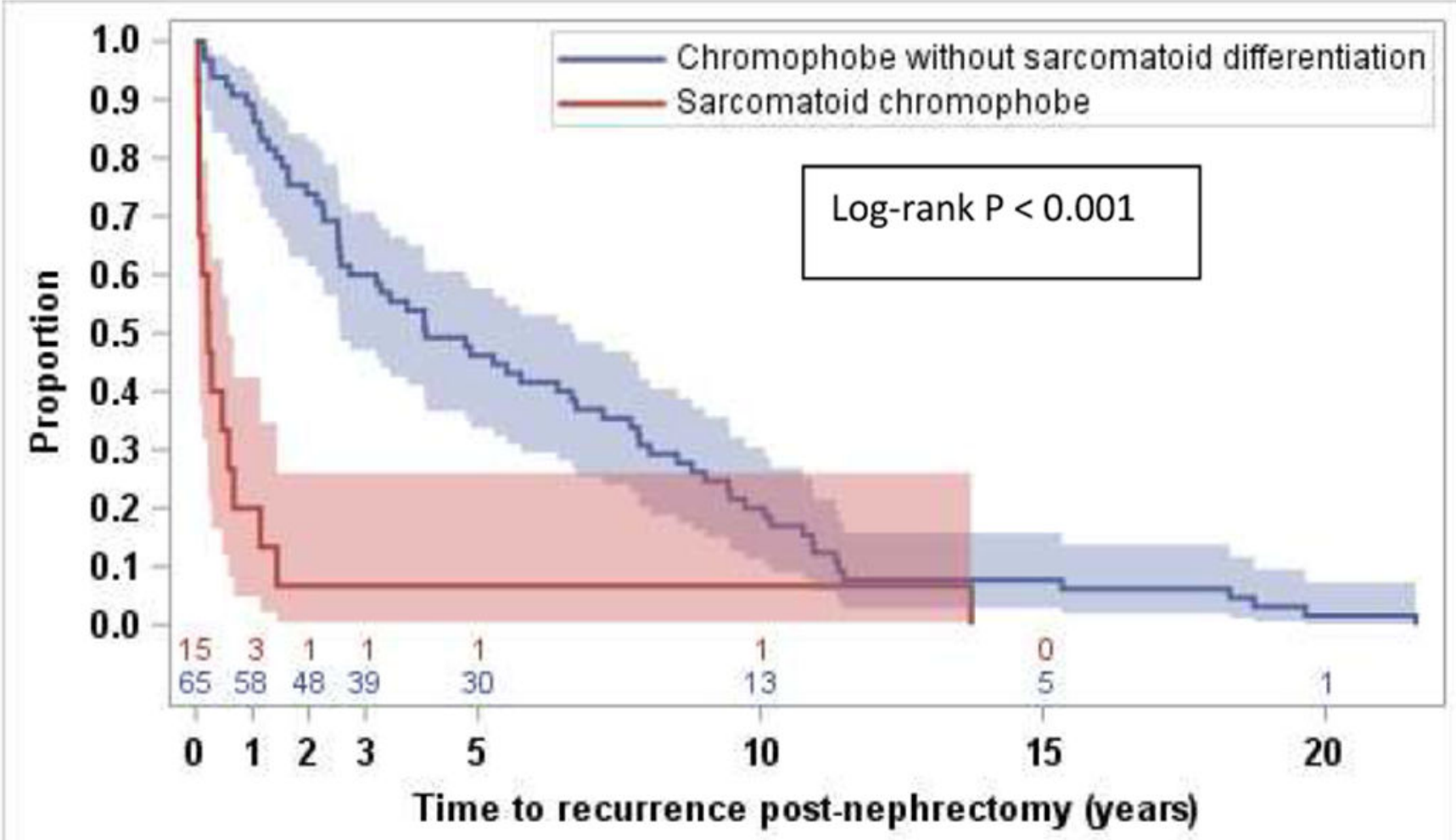

Figure 1. Time to recurrence (TTR) post nephrectomy with metastatic disease.

Median TTR with metastatic disease for patients who had initially presented with localized disease and underwent nephrectomy in curative intent was significantly longer for 65 patients without sarcomatoid differentiation compared to 15 patients with sarcomatoid differentiation (48.8 months [95\% CI: 30.8, 80.7] vs. 2.7 months [95\% CI: $0.7,6.9]$, logrank $\mathrm{p}<0.001)$ 


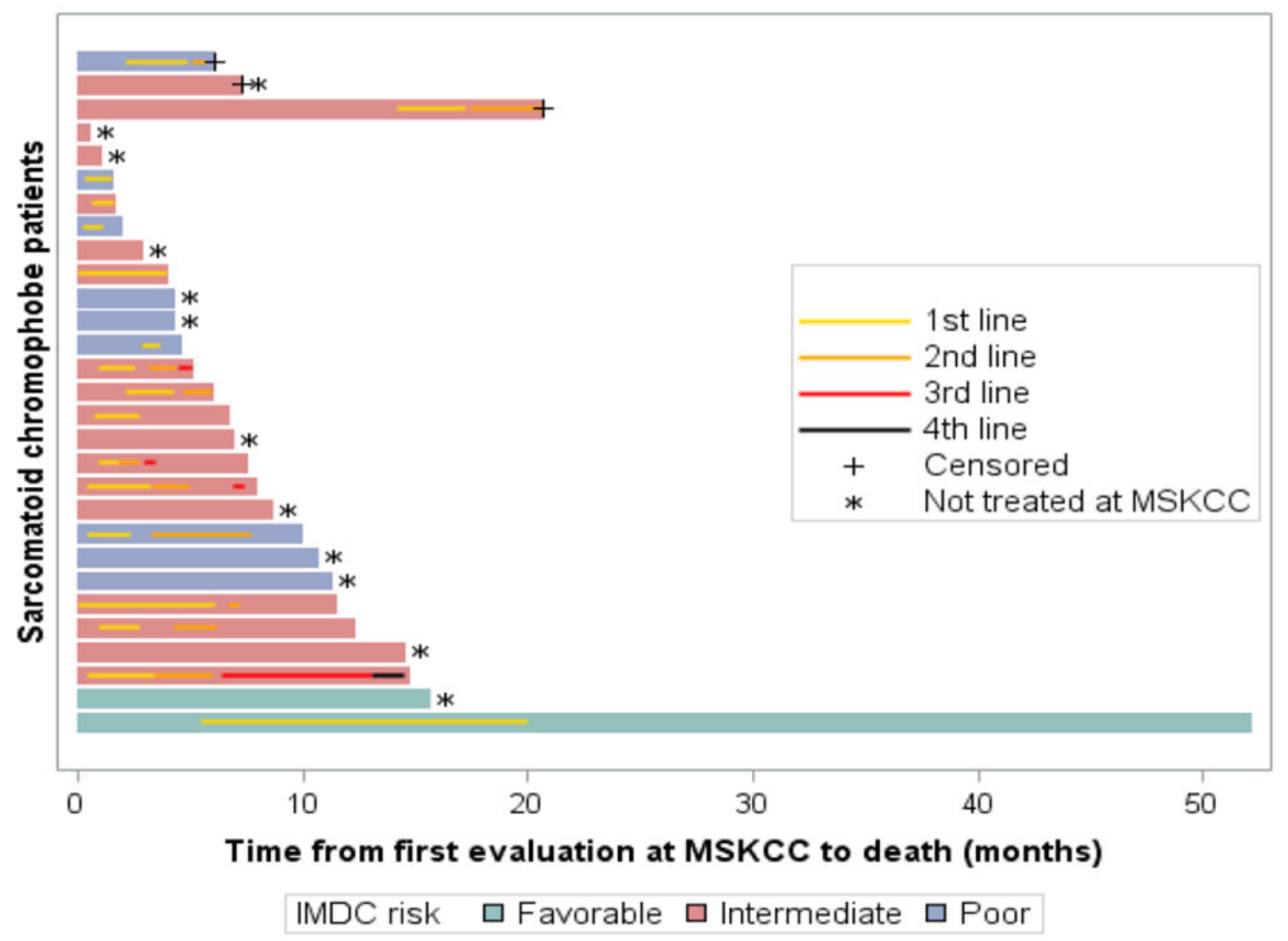

Figure 2.

Swimmer Plot illustrating treatment duration and survival time by patient for 29 sarcomatoid chromophobe renal cell carcinoma patients ( 1 censored patient was alive at the time of analysis) 


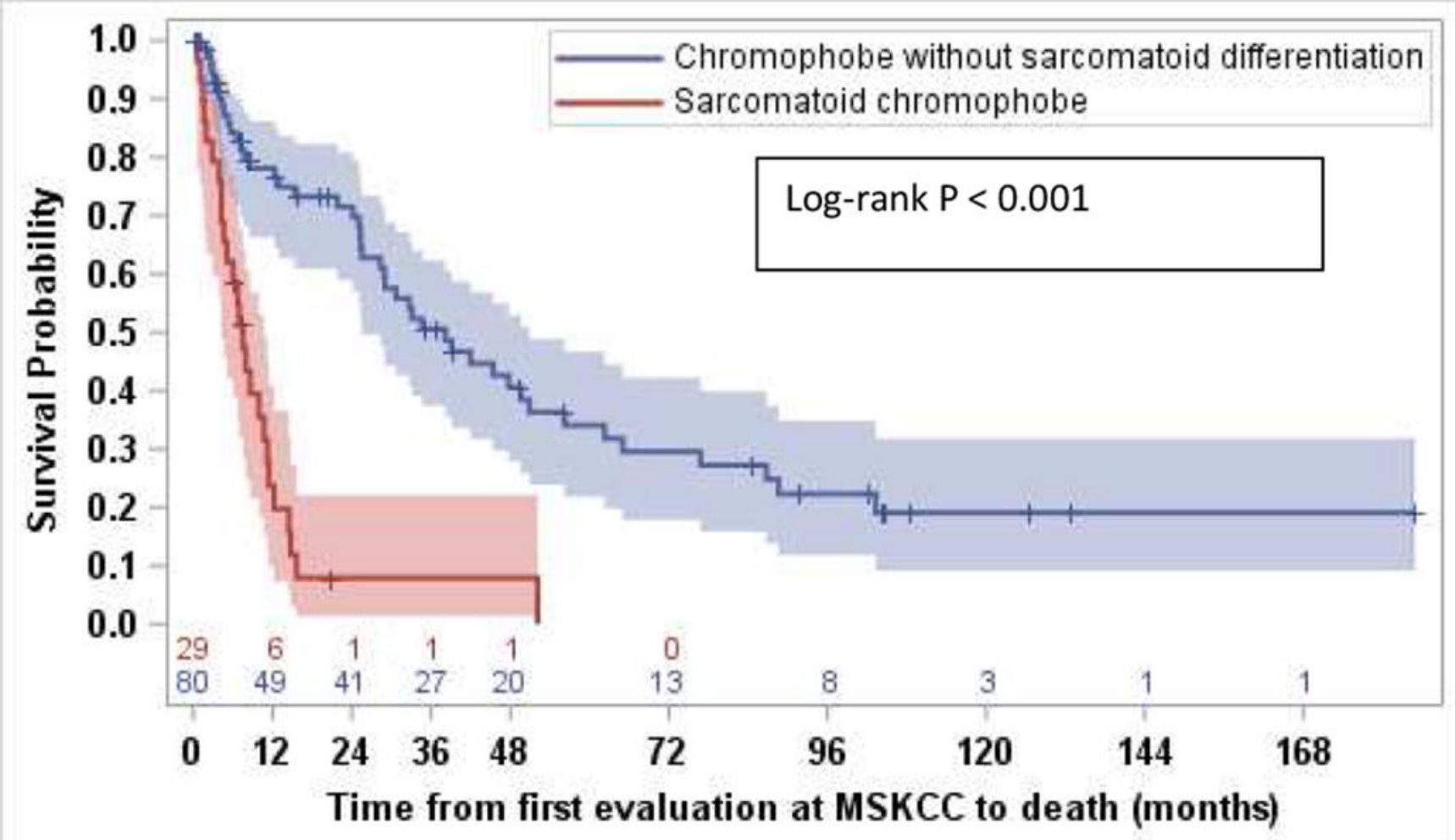

Figure 3.

Kaplan Meier curve comparing overall survival for patients with metastatic chromophobe renal cell carcinoma (chRCC) without sarcomatoid features compared to metastatic ChRCC with sarcomatoid features (38.0 months vs.7.5 months, HR 4.7, [95\% CI: 2.7, 8.2], log-rank $\mathrm{p}<0.001)$ 


\begin{tabular}{|l|l|l|l|l|l|l|l|l|l|l|l|l|l|l|l|l|l|l|l|l|l|l|}
\hline Mutation & & & & & & & & & & & & & & & & & & & & & & \\
\hline TP53 (64\%) & & & & & & & & & & & & & & & & & & & & \\
\hline PTEN (45\%) & & & & & & & & & & & & & & & & & & & & \\
\hline TERT (14\%) & & & & & & & & & & & & & & & & & & & & \\
\hline NOTCH1 (9\%) & & & & & & & & & & & & & & & & & & & & \\
\hline FOX01 (9\%) & & & & & & & & & & & & & & & & & & & \\
\hline KMT2D (9\%) & & & & & & & & & & & & & & & & & & & \\
\hline ATM (9\%) & & & & & & & & & & & & & & & & & \\
\hline
\end{tabular}

\begin{tabular}{|l|l|}
\hline Sarcomatoid & \\
\hline Non-Sarcomatoid & \\
\hline Nonsense & \\
\hline Missense & \\
\hline Promoter & \\
\hline Indel & \\
\hline Splice site & \\
\hline Fusion & \\
\hline Multiple variant & \\
\hline No alteration & \\
\hline
\end{tabular}

Figure 4.

Oncoprint illustrating most common oncogenomic changes detected by next generation sequencing using MSK- IMPACT analysis across 22 patients with columns representing individual patients 


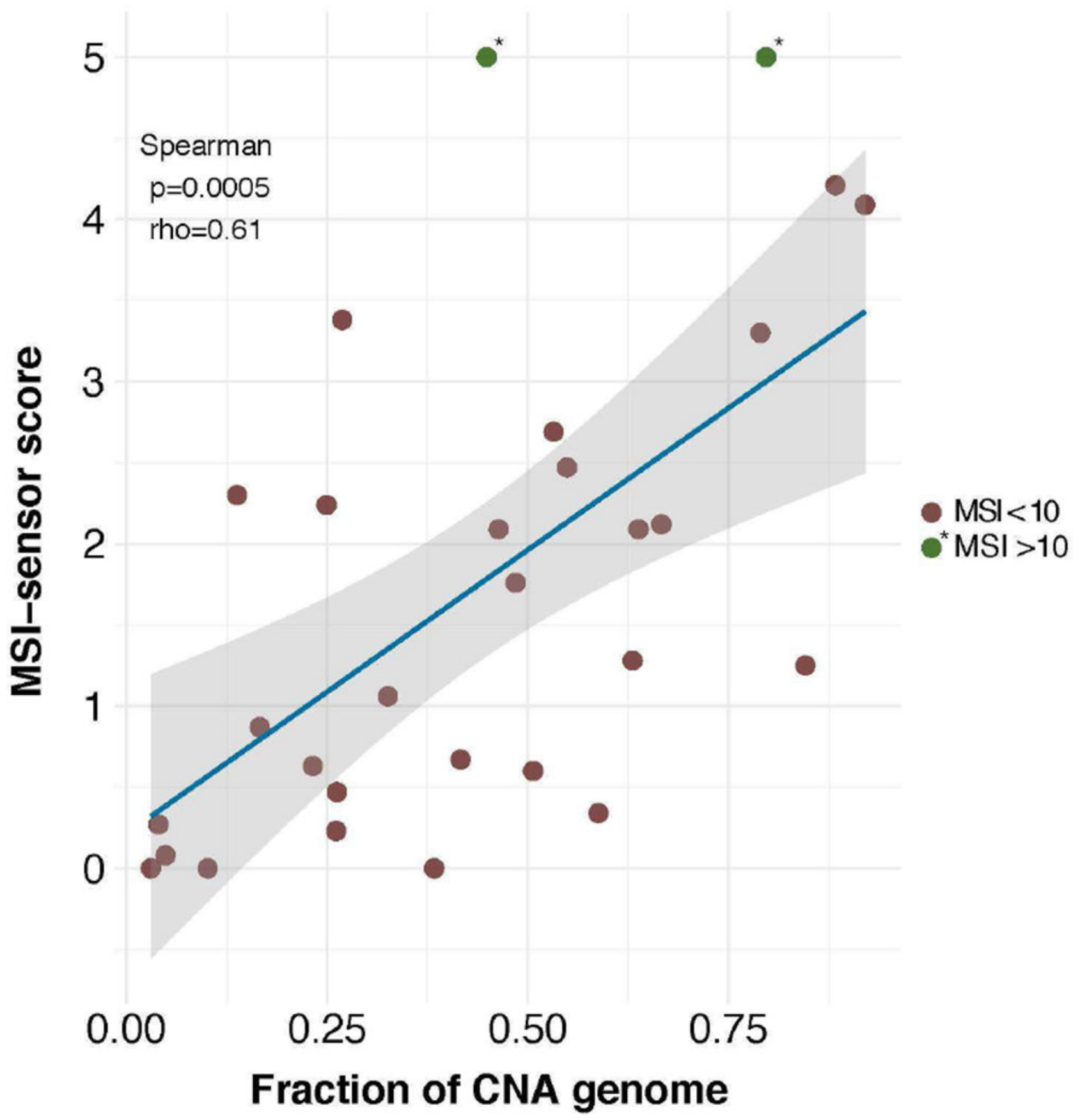

Figure 5.

Spearman rank correlation test evaluating the association between the fraction of copynumber altered genome (FCNAg) and MSI-sensor scores showing strong association between both. 
Table 1.

Baseline characteristics of 109 patients with metastatic chromophobe RCC

\begin{tabular}{|c|c|c|c|c|}
\hline & Entire cohort $(\mathrm{N}=109)$ & Sarcomatoid $(\mathbf{N}=29)$ & Non-sarcomatoid ( $\mathbf{N = 8 0 )}$ & $\begin{array}{c}\text { P-value } \\
\text { (Fisher's exact } \\
\text { test) }\end{array}$ \\
\hline Age at diagnosis (years) - median (range) & $58(40,84)$ & $53(40,77)$ & $59(40,84)$ & 0.12 \\
\hline Male gender & $60(55 \%)$ & $17(59 \%)$ & $43(54 \%)$ & 0.65 \\
\hline Diagnosed with de novo metastatic disease & $29(27 \%)$ & $14(48 \%)$ & $15(19 \%)$ & 0.002 \\
\hline IMDC risk & & & & $<0.001$ \\
\hline Favorable & $42(42 \%)$ & $2(7 \%)$ & $40(56 \%)$ & \\
\hline Intermediate & $42(42 \%)$ & $18(62 \%)$ & $24(34 \%)$ & \\
\hline Poor & $16(16 \%)$ & $9(31 \%)$ & $7(10 \%)$ & \\
\hline Nephrectomy & & & & 0.002 \\
\hline Yes & $103(94 \%)$ & $28(97 \%)$ & $75(94 \%)$ & \\
\hline No & $6(6 \%)$ & $1(3 \%)$ & $5(6 \%)$ & \\
\hline Primary tumor size $\mathrm{cm}$-median(range) & $10.5(1.9-23)$ & $10.95(6.8-21)$ & $10.25(1.9-23)$ & \\
\hline Symptoms & & & & $<0.001$ \\
\hline Asymptomatic & $40(37 \%)$ & $4(14 \%)$ & $36(45 \%)$ & \\
\hline Localized & $47(43 \%)$ & $11(38 \%)$ & $36(45 \%)$ & \\
\hline Systemic & $22(20 \%)$ & $14(48 \%)$ & $8(10 \%)$ & \\
\hline No. of metastatic sites & & & & 0.13 \\
\hline 1 & $61(56 \%)$ & $12(41 \%)$ & $49(61 \%)$ & \\
\hline 2 & $36(33 \%)$ & $12(41 \%)$ & $24(30 \%)$ & \\
\hline 3 & $10(9 \%)$ & $5(17 \%)$ & $5(6 \%)$ & \\
\hline \multicolumn{5}{|l|}{ Metastatic site } \\
\hline Lymph nodes & $47(43 \%)$ & $14(48 \%)$ & $33(41 \%)$ & 0.5 \\
\hline Lung & $37(34 \%)$ & $18(62 \%)$ & $19(24 \%)$ & $<0.001$ \\
\hline Bone & $34(31 \%)$ & $5(17 \%)$ & $29(36 \%)$ & 0.06 \\
\hline Liver & $30(27 \%)$ & $6(21 \%)$ & $24(30 \%)$ & 0.40 \\
\hline Peritoneal disease & $9(8 \%)$ & $1(3 \%)$ & $8(10 \%)$ & 0.40 \\
\hline Adrenal & $4(4 \%)$ & $3(10 \%)$ & $1(1 \%)$ & 0.05 \\
\hline Pancreas & $1(1 \%)$ & $1(3 \%)$ & $0(0 \%)$ & \\
\hline
\end{tabular}

Clin Genitourin Cancer. Author manuscript; available in PMC 2020 June 01. 
Table 2.

First line systemic therapy details for patients with metastatic chromophobe RCC

\begin{tabular}{ccc}
\hline Group & Number of patients & TTF (Median) \\
\hline All patients & 52 & 5.1 months (95\% CI: 2.7, 8.0) \\
\hline Non-sarcomatoid & 35 & 8.0 months (95\% CI: 5.1, 13.0) \\
Sarcomatoid & 17 & 1.8 months (95\% CI: 0.9, 2.7) \\
\hline Most common treatment categories & Number of patients & TTF (Median) \\
\hline VEGF-TKI & 31 & 4.4 months (95\% CI: 2.6, 6.0) \\
mTOR inhibitor & 7 & 6.2 months (95\% CI: 0.9, 13.0)
\end{tabular}

